\title{
Double-blind control
}

David F Marks

Potential competing interests: The author(s) declared that no potential competing interests exist.

A double-blind control is used in randomized controlled trials to prevent bias: both the investigator and the participant are prevented from knowing whether they are in the treatment or a control condition. 